Technical Paper

\title{
Dye-sensitised and Perovskite Solar Cells as Indoor Energy Harvestors
}

\author{
Kentaro Kawata $^{1 *}$, Koichi Tamaki ${ }^{1}$ and Masahide Kawaraya ${ }^{2}$ \\ ${ }^{1}$ Merck Ltd., 4084 Nakatsu, Aikawamachi, Aikogun, Kanagawa 243-0303, Japan \\ ${ }^{2}$ Research Center for Advanced Science and Technology, University of Tokyo, \\ 4-6-1 Komaba, Meguro, Tokyo 153-8904, Japan \\ kentaro.kawata@merckgroup.com
}

\begin{abstract}
The power output of dye-sensitised solar cells and Perovskite solar cells were evaluated under simulated indoor condition. Photopolymers were used to encapsulate the both types of photovoltaic devices which might not be otherwise thermally sufficiently stable. More than twice higher external quantum efficiency was confirmed than measurement under simulated sunlight whilst maintaining photovoltage, leading to up to nearly $20 \%$ power conversion efficiency.
\end{abstract}

Keywords: Photovoltaics, hole transport materials (HTM), Encapsulation

\section{Introduction}

Futurologists argue that everything in our life and in the industries will be connected via trillions of sensors to collect real-time big data [1-2]. Such smart autonomous systems will require energy harvestors distributed in the environment as power sources. The dye-sensitised solar cells (DSSC) is particularly suited because of its ability to maintain photovoltage even under dim light condition, outperforming conventional silicon based photovoltaics in power conversion efficiency (PCE). However, the thermal stability of DSSC is limited due to the use of liquid electrolyte. Therefore, UV-curable photoactive materials such as acrylate and epoxy polymers as shown in Fig. 1 (a) and (b) may be employed on top of thermoplastic resin [3].

In recent years, Perovskite solar cells (PSC's) have been also actively studied because of their appealing PCE as high as that of top efficiency silicon solar cells for outdoor applications and their provisional low cost production [4]. However, their indoor efficiencies have not yet been reported. PSC's might also have to be

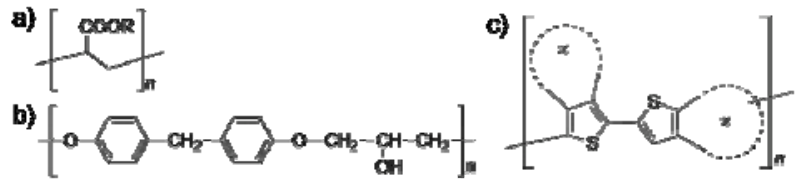

Fig. 1. Chemical structures of photogenic a) acrylate and b) epoxy polymers, and c) general structure of photoactive HTM polymers protected with photopolymers because they are known to be vulnerable to moisture stress. In PSC's photoactive polymers might be employed as a hole selective contact to facilitate charge separation from the photoactive layer because there are many electron donor polymers that assumes like p-type semiconductors. Such examples are shown in Fig. 1 (c).

In this article, we will characterise photovoltaic properties of both DSSC and PSC under indoor simulated LED illumination condition, which is generally more difficult than establishing outdoor solar simulator because of diffuse light with oblique incidence.

\section{Methods}

The DSSC's were fabricated as described in our previous report [3]. $\mathrm{TiO}_{2}$ paste was supplied from Mikuni Color, sensitising dye CYC-B11 from Tanaka Precious Metals and purely ionic liquid electrolyte for indoor use livion $^{\mathrm{TM}} 151$ from Merck. Acrylate sealing materials TB3035B from ThreeBond was used to cover the surrounding of DuPont bynel resin to retain the liquid electrolyte. The PSC's were fabricated based on a known procedure [5] using lisicon ${ }^{\circledR}$ photopolymer. Bisphenol F-type UV resin from Nagase, XNR 5570 was coated over the back side. The active area was $0.16 \mathrm{~cm}^{2}$ for the DSSC's and $0.09 \mathrm{~cm}^{2}$ for PSC. UV curing lamp from Ushio was employed for sealing both DSSC and PSC,

Current-voltage $(J-V)$ of the devices under illumination 
a)

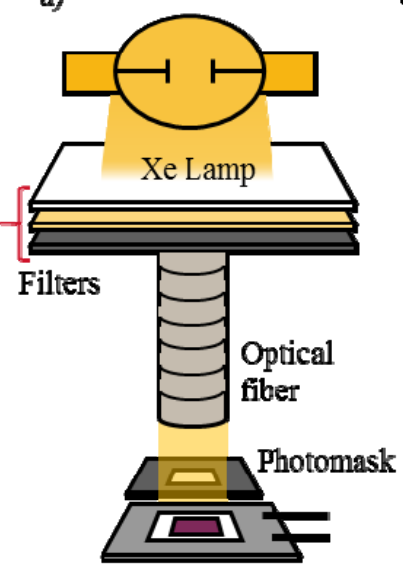

b)

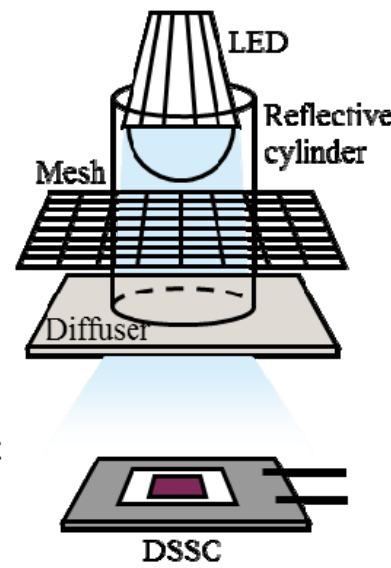

Fig. 2. Simulated light illumination of: a) solar irradiation and b) indoor light. Note that the photomask and PSC are in contact to minimise the influence of stray light.

was measured as shown in Fig. 2 and as reported previously [3]. For simulated sunlight (AM1.5) a special caution was made to make sure that all the light shined on the sample was normal incidence with using an optical filter. On the other hand, indoor light was simulated so as to insure that nearly all the light reach the sample with oblique incidence. The LED light source, Panasonic EVERLEDS LDA4N-H-E17 of $320 \mathrm{~lm}$ showed a large extent of temporal decay, and so a care had to be taken to calibrate the light intensity on time. Konica Minolta CL-500A illuminance spectrophotometer with cosine response (f2) of within 3\% was employed to accurately monitor the energy flux of incoming light with a significant fraction of diffuse light. PCE, short circuit current density $\left(J_{\mathrm{SC}}\right)$, open circuit voltage $\left(V_{\mathrm{OC}}\right)$ and fill factor (FF) were extracted based on JIS C 8913. The external quantum efficiency (EQE) was calculated to compare photocurrent under different illumination levels.

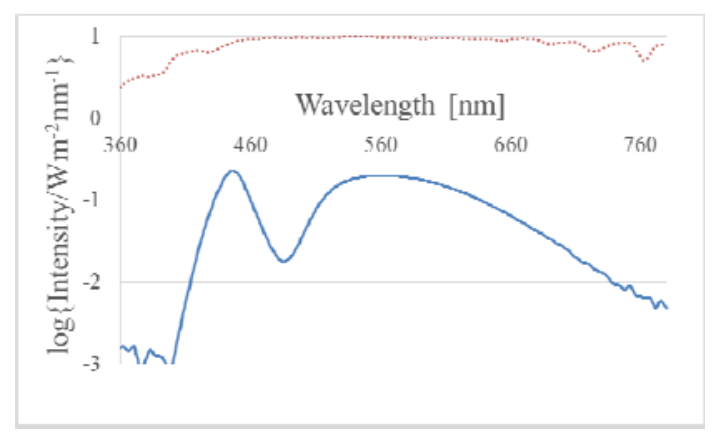

Fig. 3. Indoor light depicted in Fig. 2 as plotted with a solid line Sunny outdoor light is also shown as a dotted line.

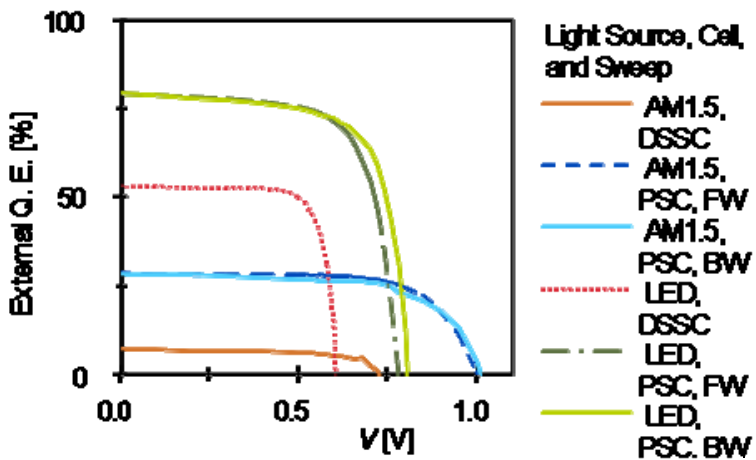

Fig. 4. EQE- $V$ curves of a DSSC and a PSC under simulated indoor and AM1.5 illumination with forward (FW) and backward (BW) sweep directions.

\section{Results}

The spectra of simulated $10^{3}$ lux indoor and $10^{5}$ lux outdoor light are shown in 3. Typical photocurrent curves as represented in EQE for each type of cells measured under the simulated light as a function of applied voltage is shown in Fig. 4. To make sure that there is no significant hysteresis in our PSC under the conditions, both sweep directions are included in the figure. The photovoltaic parameters are presented in Table 1.

Table 1. Photovoltaic characteristics of the DSSC and PSC under $1000 \mathrm{~lx}$ and AM1.5 illumination.

\begin{tabular}{ccccccc}
\hline Cell & $\begin{array}{c}\boldsymbol{J}_{\mathbf{S C}} \\
{\left[\boldsymbol{\mu} \mathbf{A} / \mathbf{c m}^{2}\right]}\end{array}$ & $\begin{array}{c}\text { EQE } \\
{[\%]}\end{array}$ & $\begin{array}{c}\boldsymbol{V}_{\mathbf{O C}} \\
{[\mathbf{V}]}\end{array}$ & $\boldsymbol{F F}$ & $\begin{array}{c}\text { Power } \\
{\left[\mu \mathbf{W} / \mathbf{c m}^{2}\right]}\end{array}$ & $\begin{array}{c}\text { PCE } \\
{[\%]}\end{array}$ \\
\hline \multicolumn{7}{c}{$A M 1.5$} \\
DSSC & 4600 & 7.2 & 0.73 & 0.63 & 2100 & 2.1 \\
PSC & 18300 & 28.6 & 1.01 & 0.67 & 12400 & 12.4 \\
& 78 & 52.8 & 0.61 & 0.78 & 37.0 & 11.2 \\
DSSC & 78000 l $L E D$ \\
PSC & 118 & 79.3 & 0.79 & 0.70 & 64.9 & 19.8 \\
\hline
\end{tabular}

\section{Discussions}

Compared with the broad solar spectrum in Fig. 3, the indoor LED illumination is composed of two peaks as represented in Fig. 3, comprising of blue LED emission centre and yellow phosphor luminescent centre. They are quite well covered with dye absorption of DSSC and rather high bandgap of organohalide lead Perovskite crystal. As a consequence, we could attain more than seven times higher external quantum efficiency for DSSC and nearly three times for PSC at the indoor condition as illustrated in Fig. 4. The larger gap observed for DSSC was because of insufficient doping of the liquid electrolyte with iodine to allow sufficient hole transport 
under outdoor condition. A large series resistance due to the liquid electrolyte manifested itself also in the reduction of FF. That did not seem to matter for PSC as the layer thickness of the photoactive polymer was much thinner [6].

On the other hand, photovoltage was significantly reduced at a low illumination level. This might be because smaller number of carrier generation could not attain quasi Fermi level high enough to minimise thermal energy loss. Overall, PCE was more than five time higher for DSSC and nearly twice higher for PSSC under the indoor condition. The PCE of up to nearly $20 \%$ for PSC is higher than any other photovoltaic devices under indoor condition as far as we know.

\section{Conclusion and outlook}

UV-curable photoactive polymers were used to encapsulate DSSC and PSC to allow for detailed measurements in the ambient condition. Overall, the performance of both DSSC and PSC under the indoor illumination condition was shown to be promising. Particularly, the quantum yield was found to be more than twice higher than outdoor condition because of better spectral matching with indoor light source. The photovoltage attained under dim light condition was found not to be greater than under bright sunshine. That could be mitigated by tuning the materials for indoor condition because the materials selected to fabricate the DSSC and PSC in this study were the ones that had been developed to maximise their performance under the standard AM1.5 measurement condition. Photoactive polymer was successfully implemented in PSC device to effectively extract hole from the interface with Perovskite layer owing to its outstanding electron blocking nature.
In order to assess the performance of photovoltaic devices under indoor conditions by more accurately taking into consideration oblique incident light, we are going to scale up the device active area by a factor of more than 100. At the same time different photoactive polymeric materials are being tested to further increase the performance.

\section{Acknowledgments}

This work was supported by New Energy and Industrial Technology Development Organization (NEDO), Japan, within the framework of the project for versatile verification on photovoltaics.

\section{References}

1. Y. Tanaka, N. Kanei, T. Horiuchi, Ricoh Technical Report in Japanese, 7 (2015) 40.

2. Guardian Angels for a smarter life "Zero Power Systems"; http://www.ga-project.eu as retrieved on the $1^{\text {st }}$ of May, 2015.

3. K. Kawata, H. Yoshizaki, T. Goto, K. Kato, S. Renker, Jpn. J. Appl. Phys., 51 (2012) 10NE06.

4. J. Burschka, N. Pellet, S.J. Moon, R. Humphry-Baker,

P. Gao, M.K. Nazeeruddin, M. Grätzel, Nature, 499 (2013) 316 .

5. K. Kawata, V.M. Burlakov, M.J. Carey, H.E. Assender, G.A.D. Briggs, A. Ruseckas, I.D.W. Samuel, Solar Energy Mat Solar Cells, 87 (2005) 715.

6. K. Kawata, K. Tamaki, F. Fabregat-Santiago, S. Ito, "Physical characterisation and failure mode analysis of Perovskite solar cells" manuscript under revision. 